\title{
Anterior Choroidal Artery Aneurysm Associated With Moyamoya Disease
}

\author{
Syunsuke Seki ${ }^{\mathrm{a}}$, Tomoya Kamide ${ }^{\mathrm{a}}$, Akira Tamase ${ }^{\mathrm{a}}$, Kentaro Mori ${ }^{\mathrm{a}}$, Yu Iida ${ }^{\mathrm{a}}$, \\ Yuichi Kawabata $^{\mathrm{b}}$, Tatsu Nakano ${ }^{\mathrm{b}}$, Motohiro Nomura ${ }^{\mathrm{a}, \mathrm{c}}$
}

\begin{abstract}
The clinical course and management of anterior choroidal artery (AChoA) aneurysms associated with moyamoya disease (MMD) have not been fully clarified. A 61-year-old man experienced dysarthria. CT revealed hemorrhage in the lateral ventricles, and an aneurysm in the left lateral ventricle. MRI performed 13 months prior to the onset showed no aneurysm. Angiography on admission demonstrated an aneurysm of about $4 \mathrm{~mm}$ in diameter on the left anterior choroidal artery. Repeated angiography on the ninth day showed enlargement of the aneurysm. However, MRI and angiography demonstrated the disappearance of the aneurysm on the 17th day. On the 38th day, superficial temporal artery-middle cerebral artery bypass was performed to decrease the hemodynamic stress on the distal arteries. Follow-up angiography showed no recurrence of the aneurysm. This is the first reported case of a distal anterior choroidal artery aneurysm, which was previously undetectable, showing enlargement followed by spontaneous disappearance. Distal aneurysms associated with MMD occasionally regress. Therefore, close radiological observation is necessary.
\end{abstract}

Keywords: Aneurysm; Anterior choroidal artery; Moyamoya disease; Intraventricular hemorrhage

\section{Introduction}

Until 2014, a total of 55 cases of intraventricular aneurysms had been reported [1-3]. Among them, 29 cases were associated with moyamoya disease (MMD). The spontaneous disappearance of aneurysms was observed in six of 55 cases of intraventricular aneurysms. As for anterior choroidal artery (AChoA)

Manuscript accepted for publication February 12, 2016

aDepartment of Neurosurgery, Yokohama Sakae Kyosai Hospital, 132 Katsura-cho, Sakae-ku, Yokohama 247-8581, Japan

bDepartment of Neurology, Yokohama Sakae Kyosai Hospital, 132 Katsuracho, Sakae-ku, Yokohama 247-8581, Japan

${ }^{\mathrm{c} C}$ Corresponding Author: Motohiro Nomura, Department of Neurosurgery, Yokohama Sakae Kyosai Hospital, 132 Katsura-cho, Sakae-ku, Yokohama 2478581, Japan. Email: nomura413jp@yahoo.co.jp

doi: http://dx.doi.org/10.14740/jnr371w aneurysms, 23 cases were associated with MMD $[1,2]$, and three showed spontaneous regression $[4,5]$. We encountered a patient with an intraventricular aneurysm on AChoA presenting with intraventricular hemorrhage. In this report, the clinical course and radiological changes of the AChoA aneurysm associated with MMD are discussed.

\section{Case Report}

A 61-year-old man transiently experienced dysarthria. He had a past history of left putaminal hemorrhage at the age of 51, and a diagnosis of MMD was made at that time. CT on admission revealed hemorrhage in the lateral ventricles (Fig. 1A). Contrast-enhanced CT (CECT) showed an aneurysm in the left lateral ventricle (Fig. 1B). MRI performed 13 months before admission in another hospital showed no aneurysm (Fig. 2A). Three-dimensional CT angiography (3D-CTA), MRI, and angiography demonstrated an aneurysm on the left $\mathrm{AChoA}$ of about $4 \mathrm{~mm}$ in diameter (Figs. 1C, 2B, and 3A). Occlusion of the bilateral internal carotid arteries and moyamoya vessels were also observed. On the fourth day, ventriculo-external drainage was performed for hydrocephalus. Follow-up angiography on the ninth day (Fig. 3B) showed enlargement of the aneurysm to a diameter of $6 \mathrm{~mm}$. Therefore, direct surgery for the aneurysm via a transcortical approach was planned. However, on the 17th day, the aneurysm had disappeared on both MRI (Fig. 2C) and angiography (Fig. 3C). The aneurysm was considered to have thrombosed spontaneously. On the 38th day, superficial temporal artery (STA)-middle cerebral artery (MCA) bypass was performed to decrease the homodynamic stress on the distal arteries. His post-operative course was uneventful. He was discharged without deficit on the 62nd day. MRI obtained 8 months after the onset showed no recurrence of the aneurysm (Fig. 2D). Angiography obtained 13 months after the onset showed no recurrence of the aneurysm (Fig. 3D).

\section{Discussion}

An aneurysm in the lateral ventricle is rare, and the incidence among intracranial aneurysms is about $0.1 \%$ [6]. The parent vessels of intraventricular aneurysms in MMD are most frequently the anterior or posterior choroidal artery [7]. Our case involved an aneurysm of the distal AChoA associated with MMD, which 

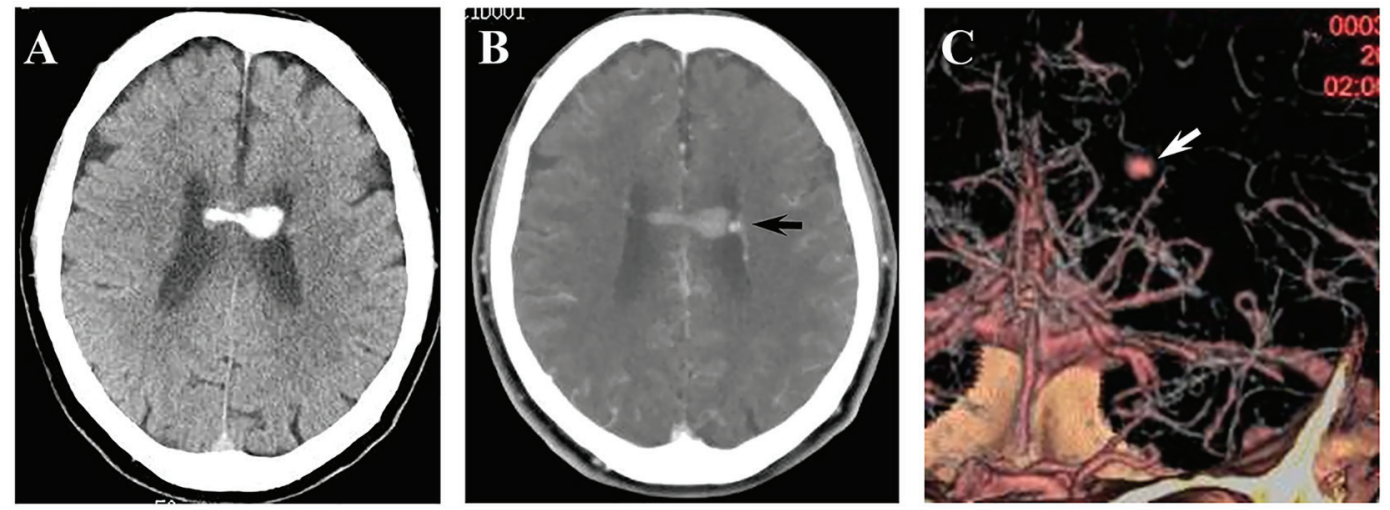

Figure 1. (A) CT on admission showing intraventricular hemorrhage. (B) CECT showing an enhanced aneurysm in the left lateral ventricle (arrow). (C) 3D-CTA showing an aneurysm on the left AChoA (arrow).

ruptured and showed intraventricular hemorrhage. To our knowledge, the present patient is the fourth reported case of an AChoA aneurysm with MMD, which showed spontaneous regression. Furthermore, this is the first case in which the absence of an aneurysm was confirmed radiologically before rupture.

Konishi et al [4] classified aneurysms associated with MMD into two groups: aneurysms located on arteries of the circle of Willis or moyamoya vessels. Most aneurysms on the circle of Willis are true ones and they do not spontaneously regress. On the other hand, peripheral artery aneurysms are considered to be pseudoaneurysms secondary to rupture of the fragile vessels, and such aneurysms sometimes disappear [8].
Konishi et al [4] reported seven patients with aneurysms associated with MMD. Among them, two cases of an AChoA aneurysm spontaneously disappeared. The disappearance of the aneurysm might have been due to vasospasm, and secondary thrombosis. In our case, the aneurysm was not demonstrated on MRI 13 months prior to the onset. During the next 13 months, the affected AChoA may have been hemodynamically stressed, and the peripheral portion finally ruptured, followed by pseudoaneurysm formation. The small aneurysm detected at onset might have been a pseudoaneurysm which showed transient enlargement and then spontaneous regression.

Treatment of a distal AChoA aneurysm is difficult due
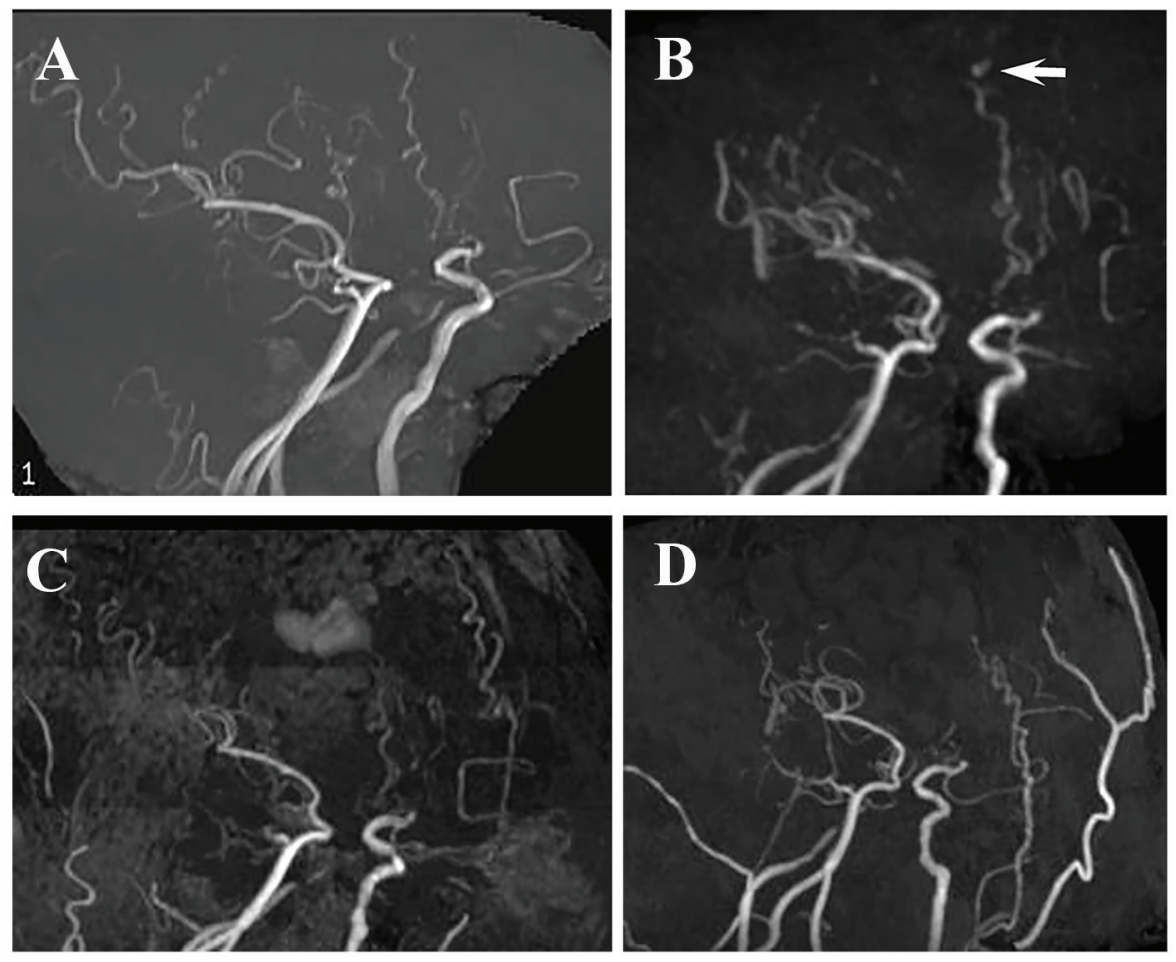

Figure 2. (A) MRI obtained 13 months before admission showing no aneurysm. (B) MRI on admission showing an aneurysm on AChoA (arrow). (C) MRI on the 17th day showing disappearance of the AChoA aneurysm. (D) MRA obtained 8 months after surgery, showing no recurrence of the aneurysm. 

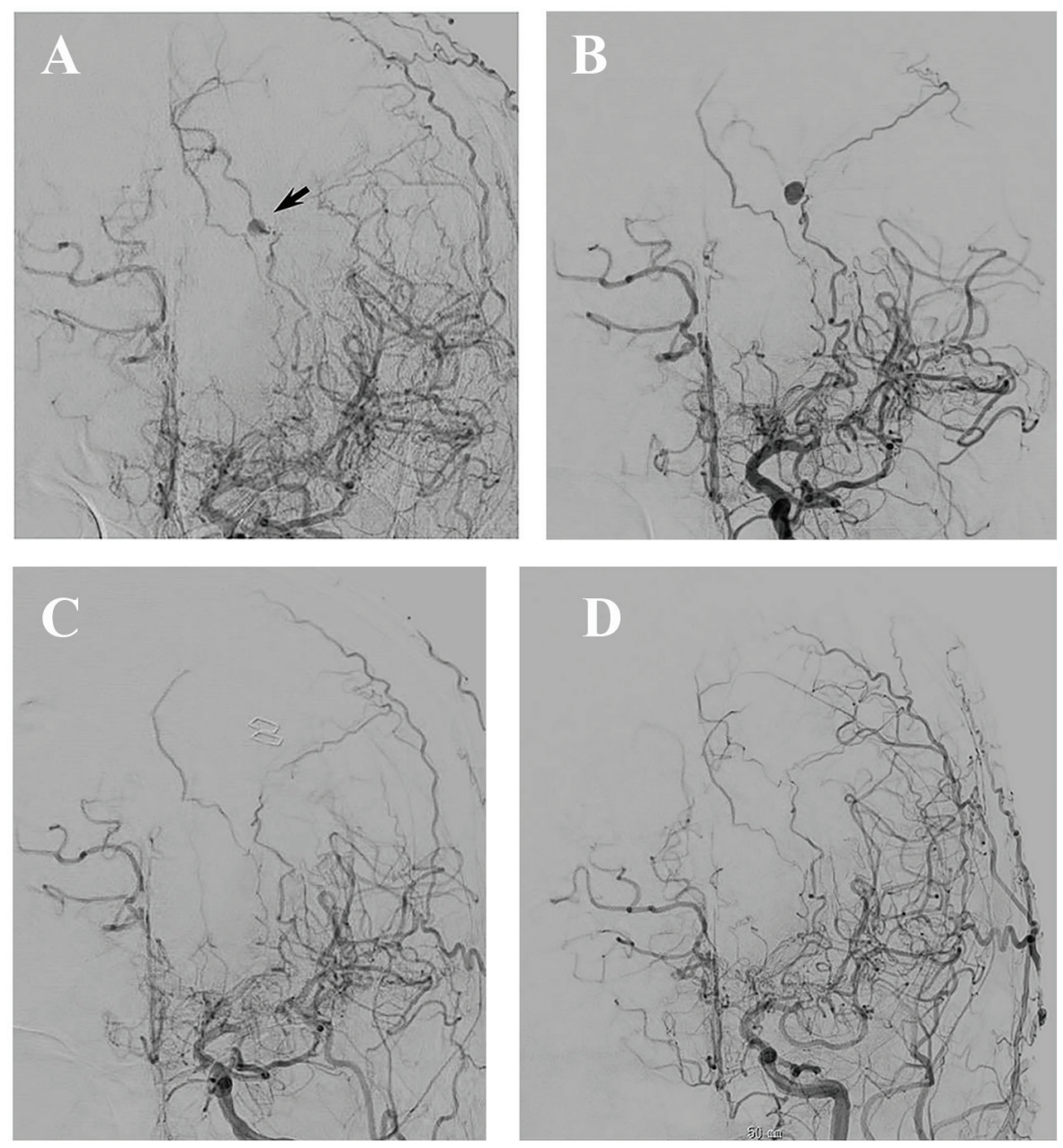

Figure 3. (A) Angiography on admission showing an aneurysm of $4 \mathrm{~mm}$ in diameter on the left AChoA (arrow). (B) Angiography on the ninth day showing enlargement of the aneurysm. (C) Angiography on the 17th day showing disappearance of the aneurysm. (D) Angiography obtained 13 months after onset showing patency of the bypass and no opacification of the aneurysm.

to its deep location [9]. Kawai et al [10] mentioned that radical treatment is necessary for some cases of ruptured distal AChoA aneurysms. On the other hand, some authors recommended the conservative management of MMD-associated aneurysms arising from choroidal arteries [8]. Regarding surgery for revascularization, Kuroda et al [5] reported three patients with peripheral artery aneurysms with MMD who were treated by STA-MCA bypass and concluded that surgical revascularization improved the cerebral circulation and decreased hemodynamic stress on collateral vessels, and finally resulted in obliteration of the peripheral artery aneurysms. Although the necessity of STA-MCA bypass for patients with aneurysm regression is not clear, we performed bypass surgery. We think that bypass surgery may be effective to prevent future hemorrhage from fragile peripheral arteries.

\section{Conflicts of Interest}

All authors declared no conflicts of interest.

\section{References}

1. Murakami Y, Sato T, Tamura T, Kyozuka H, Yasuda S, Nomura Y, Isosu T, et al. [A case of newly diagnosed moyamoya disease in a pregnant patient with a ruptured aneurysm of the distal anterior choroidal artery embolized using N-butyl cyanoacrylate]. No Shinkei Geka. 2014;42(10):961-966.

2. Okamura A, Kawamoto Y, Sakoda E, Murakami T, Hara T, Okazaki T, Kiura Y, et al. [Selective coil embolization of a distal anterior choroidal artery aneurysm associated with moyamoya disease: a case report]. No Shinkei Geka. 2014;42(5):437-444.

3. Yuan Z, Woha Z, Weiming X. Intraventricular aneurysms: case reports and review of the literature. Clin Neurol Neurosurg. 2013;115(1):57-64.

4. Konishi Y, Kadowaki C, Hara M, Takeuchi K. Aneurysms associated with moyamoya disease. Neurosurgery. 1985;16(4):484-491.

5. Kuroda S, Houkin K, Kamiyama H, Abe H. Effects of 
surgical revascularization on peripheral artery aneurysms in moyamoya disease: report of three cases. Neurosurgery. 2001;49(2):463-467; discussion 467-468.

6. Ungersbock K, Perneczky A. Intraventricular aneurysm of the medial posterior choroid artery clipped via the contralateral transcallosal approach. Acta Neurochir (Wien). 1986;82(1-2):24-27.

7. He K, Zhu W, Chen L, Mao Y. Management of distal choroidal artery aneurysms in patients with moyamoya disease: report of three cases and review of the literature. World J Surg Oncol. 2013;11(1):187.
8. Hamada J, Hashimoto N, Tsukahara T. Moyamoya disease with repeated intraventricular hemorrhage due to aneurysm rupture. Report of two cases. J Neurosurg. 1994;80(2):328-331.

9. Leveque $\mathrm{M}$, McLaughlin N, Laroche M, Bojanowski MW. Endoscopic treatment of distal choroidal artery aneurysm. J Neurosurg. 2011;114(1):116-119.

10. Kawai K, Narita K, Nakayama H, Tamura A. Ventricular hemorrhage at an early stage of moyamoya disease case report. Neurol Med Chir (Tokyo). 1997;37(2):184187. 